\title{
An efficient method for strongly correlated electrons in one dimension
}

\author{
Ion Mitxelena ${ }^{1}$ and Mario Piris ${ }^{1,2}$ \\ ${ }^{1}$ Kimika Fakultatea, Euskal Herriko Unibertsitatea (UPV/EHU) and Donostia \\ International Physics Center (DIPC), P.K. 1072, 20080 Donostia, Euskadi, Spain. \\ ${ }^{2}$ Basque Foundation for Science (IKERBASQUE), 48013 Bilbao, Euskadi, Spain.
}

\begin{abstract}
The one-particle reduced density matrix functional theory in its natural orbital functional (NOF) version is used to study strongly correlated electrons. We show the ability of the Piris NOF 7 (PNOF7) to describe non-dynamic correlation effects in one-dimensional (1D) systems. An extensive study of 1D systems that includes Hydrogen $(\mathrm{H})$ chains and the 1D Hubbard model with periodic boundary conditions is provided. Different filling situations and large sizes with up to 122 electrons are considered. Compared to quasi-exact results, PNOF7 is accurate in different correlation regimes for the 1D Hubbard model even away from the half-filling, and maintains its accuracy when the system size increases. The symmetric and asymmetric dissociations of the linear $\mathrm{H}$ chain composed of 50 atoms are described to remark the importance of long-range interactions in presence of strong correlation effects. Our results compare remarkably well with those obtained at the density-matrix renormalization group level of theory.
\end{abstract}

Keywords: Strong Electron Correlation, Hubbard model, Hydrogen chain, Dissociation, Reduced Density Matrix, Natural Orbital Functional

\section{INTRODUCTION}

One-dimensional (1D) many-electron systems remain a non-trivial problem for electronic structure methods. Density functional theory in its conventional local or semilocal approximations is not able to provide a correct description of correlated insulators [1], configuration interaction methods cannot deal with too large systems, and coupled cluster singles and doubles with perturbative triples $(\operatorname{CCSD}(\mathrm{T}))$ shows instabilities at large interatomic distances in 1D chains of Hydrogen (H) atoms [2]. Recently, significant progress has been made in lattice density functional theory [3]. Nevertheless, the densitymatrix renormalization group (DMRG) algorithm [4] remains the most accurate method for studying $1 \mathrm{D}$ systems, including gapless chains [5, 6]. Consequently, it will be employed as benchmark in this work.

The electronic wavefunction is taken as a linear combination of geminal functions to have a non-factorial scaling. In this context, variational Monte Carlo calculations using a Jastrow-antisymmetrized geminal power wavefunction has recently been used [7] to successfully investigate periodic 1D H chains. Another approach based on geminal expansions is the antisymmetric product of 1reference-orbital geminals (AP1roG). The optimized orbital version of AP1roG (OO-AP1roG) has proven [8] to be a reliable method for strongly correlated 1D systems, such as the 1D Hubbard model with periodic boundary conditions, as well as for metallic and molecular $\mathrm{H}$ rings. Nevertheless, it has recently been shown [9] that contributions from singly occupied states are important in the strong correlation limit, so OO-AP1roG needs to include open-shell configurations to accurately describe the $U / t \rightarrow \infty$ limit in the 1D Hubbard model and the disso- ciation limit in $\mathrm{H}$ chains.

The natural orbital functional theory (NOFT) [10, 11] constitutes an alternative to highly correlated methods. The energy is expressed in terms of natural orbitals (NOs) and their occupation numbers (ONs), so that from the outset NOFT correctly handles the multiconfigurational character inherent in strongly correlated systems. A route for the construction of an approximate natural orbital functional (NOF) involves the employment of necessary $N$-representability conditions for the twoparticle reduced density matrix (2RDM) [12]. Appropriate $2 \mathrm{RDM}$ reconstructions have led to different implementations known in the literature as PNOFi $(i=1-7)$ [13, 14].

The electron pairing approach came to the NOFT with the proposal of PNOF5 [15]. The latter is closely related to geminal approaches, since it corresponds to an antisymmetrized product of strongly orthogonal geminals 16]. PNOF5 draws a system of $N$ electrons as independent electron pairs providing a good description of the intrapair electron correlation, but lacks the correlation between pairs. Consequently, a bad description of the strong correlation limit is obtained [17]. To introduce interpair electron correlation effects in singlet states, PNOF7 was proposed [14, 17], namely,

$$
E=\sum_{g=1}^{N / 2} E_{g}+\sum_{f \neq g}^{N / 2} E_{f g}
$$

where

$$
\begin{gathered}
E_{g}=2 \sum_{p \in \Omega_{g}} n_{p} \mathcal{H}_{p p}+\sum_{q, p \in \Omega_{g}} \Pi_{q p} \mathcal{L}_{p q} \\
\Pi_{q p}=\left\{\begin{array}{cc}
\sqrt{n_{q} n_{p}}, & q=p \text { or } q, p>\frac{N}{2} \\
-\sqrt{n_{q} n_{p}}, & q=g \text { or } p=g
\end{array}\right.
\end{gathered}
$$




$$
\begin{gathered}
E_{f g}=\sum_{p \in \Omega_{f}} \sum_{q \in \Omega_{g}}\left[n_{q} n_{p}\left(2 \mathcal{J}_{p q}-\mathcal{K}_{p q}\right)-\Phi_{q} \Phi_{p} \mathcal{L}_{p q}\right] \\
\Phi_{p}=\sqrt{n_{p}\left(1-n_{p}\right)} .
\end{gathered}
$$

$n_{p}$ stands for the ON of the spatial NO $|p\rangle$. $\mathcal{H}_{p p}$ denotes the diagonal elements of the one-particle part of the Hamiltonian involving the kinetic energy and the external potential operators. $\mathcal{J}_{p q}$ and $\mathcal{K}_{p q}$ refer to the usual Coulomb and exchange integrals, $\langle p q \mid p q\rangle$ and $\langle p q \mid q p\rangle$ respectively, whereas $\mathcal{L}_{p q}$ denotes the exchange-timeinversion integral $\langle p p \mid q q\rangle$.

The orbital space is divided into $N / 2$ mutually disjoint subspaces $\Omega_{g}$, so that $\sum_{p \in \Omega_{g}} n_{p}=1$. Taking into account the spin, each $\Omega_{g}$ contains solely an electron pair, and the normalization condition for the one-particle reduced density matrix (1RDM) is automatically fulfilled: $2 \sum_{p} n_{p}=N$. Restriction of the ONs to the range $0 \leq n_{p} \leq 1$ represents a necessary and sufficient condition for ensemble $N$-representability of the 1RDM [18]. It should be noted that $E_{g}$ reduces to the NOF obtained from a two-electron singlet wavefunction, so the first term of PNOF7 accurately describes the sum of electron-pair energies. The second term correlates the motion of the electrons in different pairs with parallel and opposite spins. For the latter, the particle-hole symmetry is explicitly considered through $\Phi_{p}$ in the $\mathcal{L}$-term. This resembles the original formulation of Bardeen, Cooper and Schrieffer (BCS) [19], which uses these types of interactions for all orbitals. The BCS method is one of the best mean-field approaches to the Hubbard model with attractive interactions [20], but underestimates the correlation effects in systems with repulsive Hamiltonians [21]. For the latter, recent studies [17, 22, 23] suggest that PNOF7 could correctly recover the strong correlation limit. In this paper, we provide an extensive study of $\mathrm{H}$ chains composed of 50 atoms and the 1D Hubbard model in many filling situations, sizes, and correlation regimes.

The solution is established by optimizing the energy (13) with respect to the ONs and to the NOs, separately. The conjugate gradient method is used to perform the optimization of the energy with respect to auxiliary variables that enforce automatically the $N$-representability bounds of the 1RDM. The self-consistent procedure proposed in [24] yields the NOs by an iterative diagonalization procedure, in which orbitals are not constrained to remain fixed along the orbital optimization process. All calculations have been carried out using the DoNOF code developed in our group.

First, we show the ability of PNOF7 to describe the 1D Hubbard model. The latter has the advantage of being extremely simple and is a useful tool for benchmarking [25]. The 1D Hubbard Hamiltonian reads as

$$
H=-t \sum_{\left\langle r, r^{\prime}\right\rangle, \sigma}\left(a_{r, \sigma}^{\dagger} a_{r^{\prime}, \sigma}+a_{r^{\prime}, \sigma}^{\dagger} a_{r, \sigma}\right)+U \sum_{r} n_{r, \alpha} n_{r, \beta}
$$

where $\left\langle r, r^{\prime}\right\rangle$ indicates only near-neighbors hopping between the sites $r$ and $r^{\prime} . t>0$ is the hopping parameter

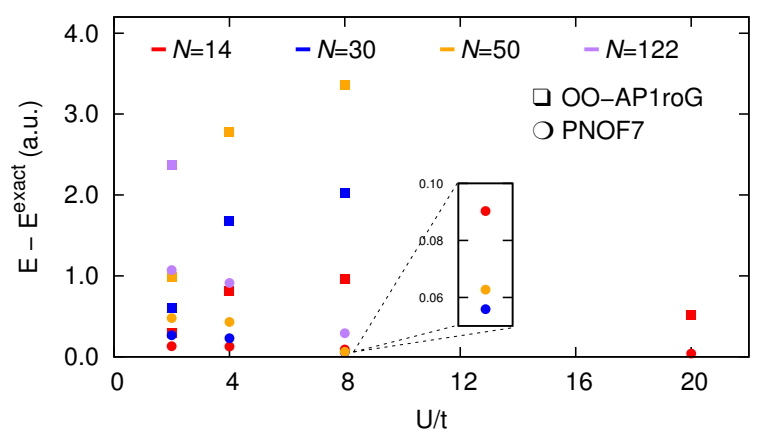

Figure 1: Energy differences (a.u.) with respect to the exact results for the 1D Hubbard model at half-filling with periodic boundary conditions. OO-AP1roG and exact data from [8, 9]. For $\mathrm{U} / \mathrm{t}=20$, only the result is reported for $N=14$.

analogous to the kinetic energy, and $U$ is the electronelectron on-site interaction parameter. $\sigma=\alpha, \beta$ stands for the spin. $a_{r, \sigma}^{\dagger}\left(a_{r, \sigma}\right)$ is the creation (annihilation) operator, so $n_{r, \sigma}=a_{r, \sigma}^{\dagger} a_{r, \sigma}$ gives the number of electrons on site $r$ with spin $\sigma$.

Let us restrict to the repulsive Hubbard model, hence $U$ is always positive. $U / t$ is used as a dimensionless measure for the relative contribution of both terms, therefore, at $U / t \rightarrow 0$ (metallic state) the mean-field theories work well due to the lack of two-electron interactions, whereas at $U / t \rightarrow+\infty$ (insulating state) strong correlations play the dominant role keeping electrons away from each other.

In Fig. [1, we report the PNOF7 energy differences with respect to the exact results for the 1D Hubbard model at half-filling. The number of sites varies from 14 to 122 in small and intermediate correlation regimes. For comparison, OO-AP1roG results [9] have been included. The data sets used in this figure can be found in the Supplemental Material (Table III). Note that OO-AP1roG deteriorates for large systems (some errors fall out of Fig. 1), as well as for large $U / t$ values. Conversely, PNOF7 is able to hold its accuracy with respect to exact results when the system size increases. For a given system, PNOF7 converges to the exact results in the strong correlation limit.

Since the particle-hole symmetry is explicitly introduced into the functional (1] [3), PNOF7 is expected to be appropriate for the half-filling case. Now we test the performance of PNOF7 away from half-filling where the particle-hole symmetry is broken, so that inhomogeneous phases can appear [25]. The energy per site for the 1D Hubbard model is shown in Table I. We focus on the strong correlation limit, i.e., large $U / t$ values, which is particularly problematic for geminal-based theories like OO-AP1roG [9]. For reference, we use the variational 2RDM (v2RDM) with $\mathrm{P}, \mathrm{Q}$ and $\mathrm{G} N$-representability constraints values and quasi-exact results of the variational Matrix Product State (vMPS) algorithm taken 
Table I: Energy per site (a.u.) for 1D Hubbard model away from half-filling at $U / t \rightarrow 100$. Reference vMPS, v2RDM, and exact data from [26]. $N_{\text {sites }}$ and $N$ stands for the number of sites and electrons, respectively.

\begin{tabular}{cccccc}
$N_{\text {sites }}$ & $N$ & PNOF7 & vMPS & v2RDM & Exact* \\
\hline \multirow{2}{*}{20} & 12 & -0.6025 & -1.0312 & -1.2177 & -1.0008 \\
& 16 & -0.3820 & -0.4951 & -0.7860 & -0.4639 \\
\multirow{2}{*}{50} & 20 & -0.9081 & - & -1.2191 & -1.0008 \\
& 40 & -0.4444 & - & -0.7862 & -0.4671 \\
\hline
\end{tabular}

*Exact results correspond to $U / t \rightarrow \infty$.

from Ref. [26].

Table I shows that PNOF7 remains close to vMPS for $N=16$ in 20 sites chain, whereas it lacks correlation energy for $N=12$. In the case of 50 sites, PNOF7 produces accurate energies and it approaches the exact result. Consequently, PNOF7 turns out to be particularly accurate from a certain amount of electrons, from which the strong correlation limit is described successfully. It is worth noting that PNOF7 is more accurate than v2RDM when only two-particle conditions are applied. It has recently been emphasized [27, 28] that three-particle conditions are needed in v2RDM to accurately describe the strong correlation limit of the Hubbard model.

In a minimal basis set, there is only one band in 1D systems, therefore, as long as long-range interactions are negligible, a linear chain composed of $\mathrm{H}$ atoms resembles the 1D Hubbard model. Such a chain composed of $50 \mathrm{H}$ atoms is a simple prototype of strong correlation, and a challenging test [2] for non-dynamic correlation.

In order to study the effect of long-range interactions, let us show the ability of PNOF7 to describe bond-breaking processes. Fig. 2 shows the energies obtained for symmetric stretching of linear $H_{50}$ by using PNOF7, together with reference DMRG results and other well-established electronic structure methods, namely, restricted Hartree-

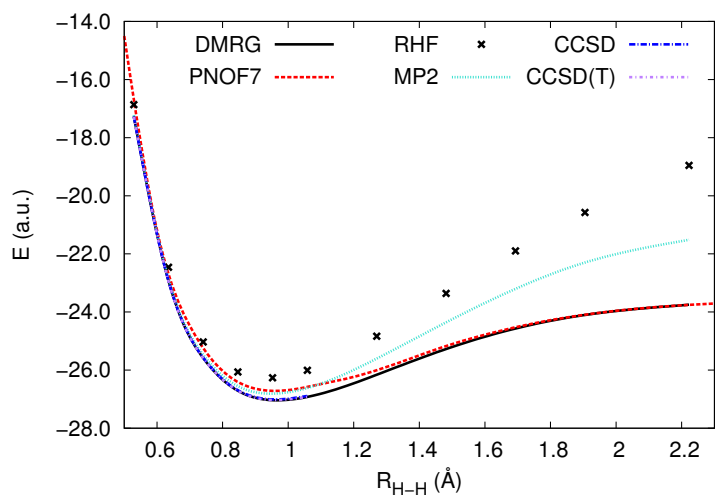

Figure 2: Symmetric dissociation of linear $H_{50}$ using the STO$6 \mathrm{G}$ basis set. RHF, MP2, CCSD, CCSD(T), and DMRG data from [2].
Table II: Equilibrium distances $\left(R_{e}\right)$ and dissociation energies $\left(D_{e}\right)$ for the symmetric dissociation of linear $H_{50}$ using the STO-6G basis set. RHF, MP2, PBE, OO-AP1roG, and DMRG data from [8].

\begin{tabular}{c|c|c|c|c|c|c} 
& RHF & MP2 & PBE & OO-AP1roG & PNOF7 & DMRG \\
\hline$R_{e}(\AA)$ & 0.940 & 0.955 & 0.971 & 0.966 & 0.976 & 0.970 \\
$D_{e}(e V)$ & 199.0 & 144.1 & 146.6 & 82.2 & 86.9 & 89.7
\end{tabular}

Fock (RHF), second-order Möller-Plesset pertubation theory (MP2), CCSD, and $\operatorname{CCSD}(\mathrm{T})$. All calculations were carried out using the STO-6G minimal basis [29]. There is an outstanding agreement between PNOF7 and DMRG along the dissociation curve, specially at large bond distances (insulating phase) as well as at short $\mathrm{H}-\mathrm{H}$ distances (metallic phase). At the equilibrium distance, PNOF7 underestimates slightly the correlation, however an inspection of spectroscopic constants (see Table II) shows that PNOF7 agrees with DMRG better than standard methods such as RHF, MP2, or the Perdew-BurkeErnzerhof (PBE) density functional. These methods fail dramatically at the dissociation limit [8] since the occupancies become strongly fractional at intermediate and long $\mathrm{H}-\mathrm{H}$ distances, a behavior that PNOF7 (see Fig. 3) and OO-AP1roG (see Fig. 4 in Ref. [8]) correctly reproduce. Non-integer occupations also make CCSD and $\operatorname{CCSD}(\mathrm{T})$ not convergent [2], so the latter can be exclusively employed in the equilibrium region. Note that OOAP1roG underestimates the equilibrium distance $\left(R_{e}\right)$ and dissociation energy $\left(D_{e}\right)$, whereas PNOF7 underestimates $D_{e}$ and yields slightly large $R_{e}$.

Fig. 4 shows the energies obtained for the asymmetric dissociation of linear $H_{50}$. It should be noted that the energy decreases monotonically from the reference state composed of equidistant $\mathrm{H}$ atoms to the set of independent $\mathrm{H}_{2}$ molecules. In the asymmetric stretching, we alternate the bond-stretching, so that half of the

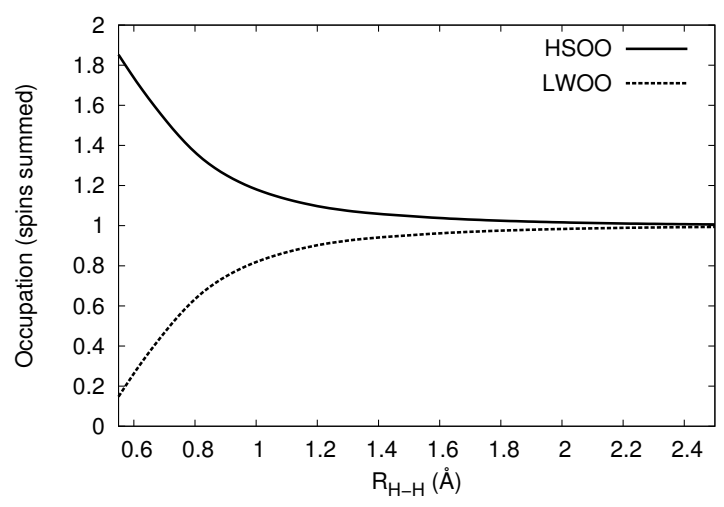

Figure 3: ONs of the highest strongly occupied NO (HSOO) and the lowest weakly occupied NO (LWOO) for the symmetric dissociation of linear $H_{50}$ at the PNOF7/STO-6G level of theory. 


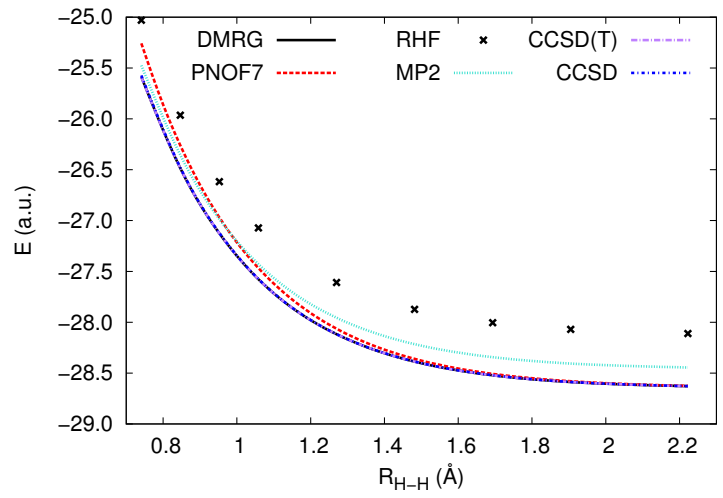

Figure 4: Asymmetric dissociation of linear $H_{50}$ using the STO-6G basis set. RHF, MP2, CCSD, CCSD(T), and DMRG data from [2].

bonds remain fixed, while the other half is stretched. In the dissociation limit, we have 25 near-independent $H_{2}$ molecules. Similar to symmetric dissociation, PNOF7 agrees with DMRG over large bond distances, whereas there are slight differences at shorter bonds.
The results obtained for $H_{50}$ chains prove that numerical accuracy of PNOF7 is comparable to that of the DMRG in many different correlation regimes. This study includes the PNOF7 in the list of highly correlated methods to study any system related to linear H chains [30].

With the present work, a step forward has been taken in the development of efficient methods for strong correlation. With a mean-field scaling, the PNOF7 approximation compares with state-of-the-art methods for describing strongly correlated electrons, e.g. DRMG, quantum Monte Carlo or complete active space configuration interaction methods, and overcomes the problems shown by similar approaches in the strong correlation limit. The present paper will have a significant impact on the development of new materials in which large unit cells are required.

Acknowledgments: Financial support comes from MCIU/AEI/FEDER, UE (PGC2018-097529-B-100) and Eusko Jaurlaritza (Ref. IT1254-19). The authors thank for technical and human support provided by IZO-SGI SGIker of UPV/EHU and European funding (ERDF and ESF). I.M. is grateful to Vice-Rectory for research of the $\mathrm{UPV} / \mathrm{EHU}$ for the Ph. D. grant (PIF//15/043).
[1] D. J. Carrascal, J. Ferrer, J. C. Smith, and K. Burke, J. Phys.: Condens. Matter 27, 393001 (2015).

[2] J. Hachman, W. Cardoen, and G. K.-L. Chan, J. Chem. Phys. 125, 144101 (2006).

[3] T. S. Müller, W. Töws, and G. M. Pastor, Computation 7, 66 (2019).

[4] S. R. White, Phys. Rev. Lett. 69, 2863 (1992).

[5] T. Papenbrock, T. Barnes, D. J. Dean, M. V. Stoitsov, and M. R. Strayer, Phys. Rev. B 68, 024416 (2003).

[6] V. O. Cheranovskii, V. V. Slavin, A. L. Tchougréeff, and R. Dronskowski, J. Phys.: Condens. Matter 31, 305601 (2019).

[7] L. Stella, C. Attaccalite, S. Sorella, and A. Rubio, Phys. Rev. B 84, 245117 (2011).

[8] K. Boguslawski, P. W. Ayers, P. Bultinck, S. D. Baerdemacker, and D. V. Neck, Phys. Rev. B 89, 201106(R) (2014).

[9] K. Boguslawski, P. Tecmer, and Ö. Legeza, Phys. Rev. B 94, 155126 (2016).

[10] M. Piris and J. M. Ugalde, Int. J. Quantum Chem. 114, 1169 (2014).

[11] K. Pernal and K. J. H. Giesbertz, Top. Curr. Chem. 368, 125 (2016).

[12] M. Piris, Int. J. Quantum Chem. 106, 1093 (2006).

[13] M. Piris, Int. J. Quantum Chem. 113, 620 (2013).

[14] M. Piris, Phys. Rev. Lett. 119, 063002 (2017).

[15] M. Piris, X. Lopez, F. Ruipérez, J. M. Matxain, and J. M. Ugalde, J. Chem. Phys. 134, 164102 (2011).

[16] M. Piris, J. M. Matxain, and X. Lopez, J. Chem. Phys. 139, 234109 (2013).

[17] I. Mitxelena, M. Rodríguez-Mayorga, and M. Piris, Eur.
Phys. J. B 91, 109 (2018).

[18] A. J. Coleman, Rev. Mod. Phys. 35, 668 (1963).

[19] J. Bardeen, L. N. Cooper, and J. R. Schrieffer, Phys. Rev. 108, 1175 (1957).

[20] M. Qin, H. Shi, and S. Zhang, Phys. Rev. B 94, 085103 (2016).

[21] T. Tsuchimochi and G. E. Scuseria, J. Chem. Phys. 131, 121102 (2009).

[22] I. Mitxelena, M. Piris, and M. A. R. Mayorga, J. Phys. Condens. Matter 29, 425602 (2017).

[23] I. Mitxelena, M. Piris, and M. Rodríguez-Mayorga, J. Phys. Condens. Matter 30, 089501 (2018).

[24] M. Piris and J. M. Ugalde, J. Comput. Chem. 30, 2078 (2009).

[25] J. P. F. and LeBlanc, A. E. Antipov, F. Becca, I. W. Bulik, G. K.-L. Chan, C.-M. Chung, Y. Deng, M. Ferrero, T. M. Henderson, C. A. Jiménez-Hoyos, et al., Phys. Rev. X 5, 1 (2015).

[26] B. Verstichel, H. van Aggelen, W. Poelmans, S. Wouters, and D. V. Neck, Comput. Theor. Chem. 1003, 12 (2013).

[27] N. C. Rubin and D. A. Mazziotti, Theor. Chem. Acc. 133, 1492 (2014).

[28] B. Verstichel, H. van Aggelen, W. Poelmans, and D. Van Neck, Phys. Rev. Lett. 108, 213001 (2012).

[29] B. P. Pritchard, D. Altarawy, B. Didier, T. D. Gibson, and T. L. Windus (2019 (in preparation)).

[30] M. Motta, D. M. Ceperley, G. K.-L. Chan, J. A. Gomez, E. Gull, S. Guo, C. A. Jiménez-Hoyos, T. N. Lan, J. Li, F. Ma, et al., Phys. Rev. X 7, 031059 (2017). 
Table III: Energies (a.u.) for the 1D Hubbard model at half-filling with periodic boundary conditions. OO-AP1roG, RHF, and exact data from Ref. [8, 9].

\begin{tabular}{cccccc}
$N_{\text {sites }}$ & $\mathrm{U} / \mathrm{t}$ & $\mathrm{RHF}$ & $\mathrm{OO}-\mathrm{AP} 1 \mathrm{roG}$ & $\mathrm{PNOF} 7$ & $\mathrm{EXACT}$ \\
\hline \multirow{4}{*}{14} & 2 & -10.9758 & -11.6627 & -11.8230 & -11.9543 \\
& 4 & -3.9758 & -7.2701 & -7.9610 & -8.0883 \\
& 8 & 10.0242 & -3.6471 & -4.5228 & -4.6131 \\
& 20 & 52.0242 & -1.4132 & -1.8932 & -1.9340 \\
\hline \multirow{2}{*}{30} & 2 & -23.2671 & -24.7779 & -25.1161 & -25.3835 \\
& 4 & -8.2671 & -15.5495 & -17.0035 & -17.2335 \\
& 8 & 21.7329 & -7.8152 & -9.78283 & -9.8387 \\
\hline \multirow{2}{*}{50} & 2 & -38.7039 & -41.2570 & -41.7650 & -42.2443 \\
& 4 & -13.7039 & -25.9154 & -28.2696 & -28.6993 \\
& 8 & 36.2961 & -13.0253 & -16.3215 & -16.3842 \\
\hline \multirow{2}{*}{122} & 2 & -94.3524 & -100.6497 & -101.9499 & -103.0211 \\
& 4 & -33.3524 & -63.2336 & -69.0861 & -70.0003 \\
& 8 & 88.6476 & -31.7817 & -39.6698 & -39.9619 \\
\hline
\end{tabular}

